Research article

\title{
Utility of the Mayo End-Stage Liver Disease (MELD) score in assessing prognosis of patients with alcoholic hepatitis Milan Sheth1, Mark Riggs ${ }^{2}$ and Tushar Patel*1
}

Address: ${ }^{1}$ Division of Gastroenterology, Scott and White Clinic, Texas A\&M University Health Science Center College of Medicine, Temple, Texas, USA and ${ }^{2}$ Division of Biostatistics, Scott and White Clinic, Texas A\&M University Health Science Center College of Medicine, Temple, Texas, USA

E-mail: Milan Sheth - msheth@swmail.sw.org; Mark Riggs - mriggs@swmail.sw.org; Tushar Patel* - tpatel@medicine.tamu.edu

${ }^{*}$ Corresponding author

Published: 22 January 2002

BMC Gastroenterology 2002, 2:2
Received: 2 December 200I

Accepted: 22 January 2002

This article is available from: http://www.biomedcentral.com/I47I-230X/2/2

(c) 2002 Sheth et al; licensee BioMed Central Ltd. Verbatim copying and redistribution of this article are permitted in any medium for any purpose, provided this notice is preserved along with the article's original URL.

\begin{abstract}
Background: Alcoholic hepatitis is characterized by acute, or acute-on-chronic hepatic failure and associated with a high mortality. Specific therapies should be considered for those at high risk of mortality. The Mayo End-Stage Liver Disease (MELD) score is a marker of disease severity and mortality in persons with chronic alcoholic liver disease. Our aims were to assess the utility of the MELD score as a predictor of short-term mortality in persons with alcoholic hepatitis.
\end{abstract}

Methods: We assessed the utility of the MELD score and compared it with the Discriminant Function (DF) as a predictor of mortality in 34 patients hospitalized with alcoholic hepatitis.

Results: The area under the curve of a receiver operating characteristic curve for the MELD score was 0.82 (confidence intervals $0.65-0.98$ ), and for the DF was 0.86 (confidence intervals $0.70-1.00$ ). However, the sensitivity and specificity in predicting 30-day mortality for a MELD score of greater than II was $86 \%$ and $81 \%$, but for a DF greater than 32 was $86 \%$ and $48 \%$ respectively. The presence of ascites and bilirubin greater than $8 \mathrm{mg} / \mathrm{dL}$ were also highly predictive of mortality with a sensitivity of $71 \%$ and a specificity of $96 \%$.

Conclusions: Alcoholic hepatitis remains associated with a high mortality in hospitalized patients. The MELD score performs as well as the DF in predicting mortality at 30 days. A MELD score of greater than II, or the presence of both ascites and an elevated bilirubin greater than $8 \mathrm{mg} / \mathrm{dL}$ should prompt consideration of specific therapeutic interventions to reduce mortality.

\section{Background}

Alcoholic hepatitis is a manifestation of alcoholic liver injury characterized by necrotizing inflammatory hepatic lesions and acute hepatic decompensation. In severe cases, alcoholic hepatitis is associated with a high mortality [1]. The treatment of alcoholic hepatitis is largely symptomatic, with emphasis on alcohol abstinence and meticulous attention to nutritional status [2]. Specific therapies such as corticosteroids and pentoxyfilline need to be consid- ered as they may reduce mortality in those patients who are at high risk of dying $[3,4]$. Thus, the identification of individuals at high risk of mortality is essential for the management of persons presenting with alcoholic hepatitis.

Persistent alcohol intake may result in collagen deposition around the central vein with central hyaline sclerosis, and eventually result in fibrotic lesions. Indeed, alcoholic 
hepatitis may be superimposed on chronic hepatic insufficiency or cirrhosis. The Mayo End Stage Liver Disease (MELD) score is becoming widely used for the prediction of mortality from end-stage liver disease and is a marker of disease severity and predictor of mortality in patients with alcoholic cirrhosis [5]. Furthermore, the individual components of the MELD score have been described as individual predictors of mortality from alcoholic hepatitis in various studies [6-8]. Thus, the MELD score may be useful as a predictor of mortality in alcoholic hepatitis.

Historically, patients with severe alcoholic hepatitis have been reported to have a 30 -day mortality as high as $50 \%$. The Discriminant Function (DF) was reported as a predictor of mortality risk based on a retrospective analysis of a severely ill subgroup of patients performed over 2 decades ago [3]. A DF score of greater than 32 identified those persons who have a greater than $50 \%$ mortality at 1 month, and who may benefit from corticosteroid therapy [3]. However, the DF requires the use of the prothrombin time (PT) which has been largely replaced by the International Normalized Ratio (INR) and is not always reported by many clinical laboratories.

Our recent clinical experience suggests that the mortality from alcoholic hepatitis may not be as high as previously reported. Improvements in critical care management, and recognition of the importance of nutritional therapy may account for these observations. However, selection bias needs to be considered since most reports of predictors of prognosis in patients with alcoholic hepatitis have been derived from patients seen at referral centers or enrolled in clinical trials. Indeed, there is a paucity of information on community-based survival or prognostic factors for alcoholic hepatitis. Thus, the aims of our study were (1) to compare the MELD score with the DF score as a predictor of short-term mortality in persons with alcoholic hepatitis, and (2) to assess prognostic factors for persons with alcoholic hepatitis seen in contemporary clinical practice.

\section{Methods}

Patients and methods

All persons hospitalized at Scott and White Memorial Hospital between Jan 01, 1997 and Dec 31, 2000 with a primary admission diagnosis of alcoholic hepatitis (ICD9 code of 571.1) were identified using an electronic medical record database. The presence of alcoholic hepatitis was confirmed on clinical, biochemical and histological grounds (where available). Demographic, clinical and laboratory data were obtained retrospectively using the electronic medical database or by chart review. Survival at 30 and 90 days following the index admission was determined by chart review or telephone follow-up for all patients.

\section{Derivation of prognostic scores}

The MELD score and modified Maddrey's Discriminant Function were calculated based on laboratory values obtained within 24 hours of admission. Only those individuals for whom all the required laboratory data were available within 24 hours of admission were included in this study. The formula used for the calculations were as follows: $M E L D$ score $=3.8^{*} \log _{e}($ total bilirubin, $\mathrm{mg} / \mathrm{dL})+$ $11.2 * \log _{e}(\mathrm{INR})+9.6 * \log _{e}($ creatinine, $\mathrm{mg} / \mathrm{dL})$; Upper or lower limits for the laboratory parameters were not applied in the derivation of the MELD score in order to obtain a continuous score. Discriminant Function $=4.6^{*}$ [patient's PT - control PT] + total bilirubin (mg/dL).

\section{Statistical analysis}

The prognostic utility of the MELD score or the DF to predict mortality at 30 days was assessed by determining the area under the curve (AUC) of a receiver operating characteristic curve. Analysis of prognostic factors was performed by first identifying the association between selected demographic, clinical or laboratory variables with 30-day mortality by univariate analysis using a chisquared test. The modeling approach involved first selecting the variable with the highest correlation coefficient with 30-day mortality. Next, the variable with the highest partial correlation with 30-day mortality was selected, holding the initial variable constant. This was repeated until none of the partial correlations were significant.

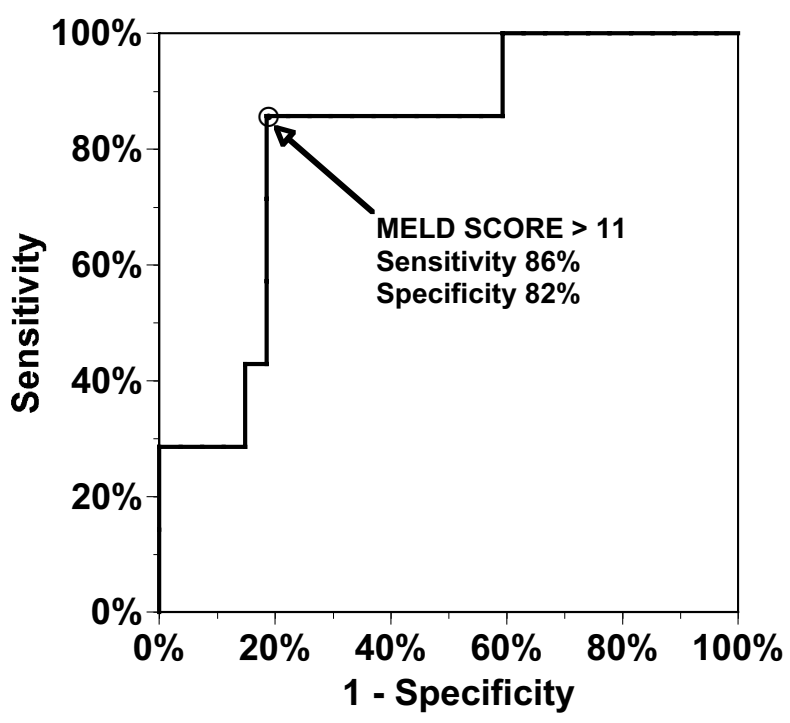

\section{Figure I}

Receiver Operating Characteristic curves for the utility of the MELD score for prediction of 30-day mortality in persons hospitalized with alcoholic hepatitis. The area under the curve (AUC) represents the predictive utility of the MELD score and is 0.82 (confidence intervals $0.65-0.98$ ). 


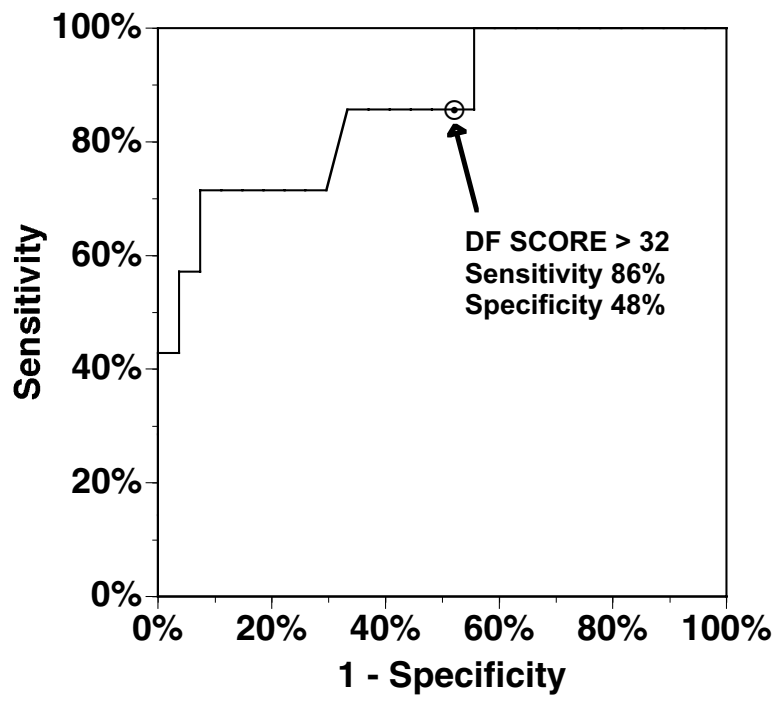

Figure 2

Receiver Operating Characteristic curves for the utility of the Discriminant Function (DF) for prediction of 30-day mortality in persons hospitalized with alcoholic hepatitis. The area under the curve (AUC) represents the predictive utility of the DF score and is 0.86 (confidence intervals $0.70-1.00$ ).

\section{Results}

Fifty-two persons were identified with an admission diagnosis ICD-9 code of 571.1. Eighteen persons did not have all the necessary laboratory tests within 24 hours of admission, and were excluded from the analysis. For the 34 patients for whom complete biochemical data were available, a diagnosis of alcoholic hepatitis was confirmed and follow-up information obtained by chart review or telephone follow-up. There were 23 men and 11 women with a mean age of 52 years. Of the 34 patients, 8 persons ( 6 men and 2 women) had a history of prior admissions for alcoholic hepatitis and the index admission was felt to represent an acute exacerbation of chronic alcoholic liver disease. The remainder had presented with acute alcoholic hepatitis. One patient had liver biopsy performed which had revealed histopathological features of steato-hepatitis. Three persons had serum antibodies to hepatitis C, but none had circulating hepatitis B surface antigen. The median length of hospital stay was 5 days (range 1 to 66 days). The overall 30-day mortality in these patients was $21 \%$, and no additional deaths occurred over the next 60 days. Thus, alcoholic hepatitis remains associated with a high short-term mortality.

The mean MELD score at admission for survivors at 30days was 4.3 , while for non-survivors was 18.7 . The predictive utility of the MELD score for prediction of mortality at 30 days was determined by generating a receiver operating characteristic curve. The area under the curve (AUC) for the MELD score was 0.82 (confidence intervals 0.65-0.98) (Fig 1). Using a cut-off of a MELD score of greater than 11 , the sensitivity and specificity of the MELD score for prediction of 30-day mortality were $86 \%$ and $82 \%$ respectively.

The DF at admission was also calculated for each person. The mean DF for survivors was 36.9 and for non-survivors 152.0. However, the AUC for the DF was 0.86 (confidence intervals 0.70-1.00) (Fig 2). Thus, the performance characteristics of the MELD score is similar to that of the DF. However, the sensitivity and specificity of a DF>32 were $86 \%$ and $48 \%$ respectively. Although current practice is to use a DF cut-off of 32, the specificity of the DF could be increased to $67 \%$ using a cut-off DF score of 42 without altering the sensitivity [9]. Thus, a MELD score of greater than 11 more accurately identified those persons with alcoholic hepatitis at high risk of short term mortality. Both the DF and the MELD score correlated poorly with the length of hospital stay (correlation coefficient 0.43 and 0.31 respectively).

We next assessed prognostic factors associated with 30day mortality amongst our patient population. A variety of prognostic factors were identified by univariate analysis (Table 1). A highly significant association was noted for serum bilirubin, INR, and the presence of ascites or encephalopathy. Of these, an elevated bilirubin greater than $8 \mathrm{mg} / \mathrm{dL}$, and the presence of ascites were highly predictive of mortality. The presence of either bilirubin or ascites has a sensitivity of $100 \%$ and specificity of $56 \%$ whereas the combination of these two factors was highly predictive of 30-day mortality, with a sensitivity of $71 \%$ and a specificity of $96 \%$ (Table 2).

\section{Discussion}

Both the MELD score and the DF score at admission are very good predictors of mortality at 30 days. Although a MELD score of $>11$ was more sensitive than a DF of $>32$, the differences were small, and unlikely to confer greater advantage in clinical use. Indeed, the performance characteristics of the two tests based on analysis of their receiver operating characteristic curves are quite similar. Thus, this study validates the use of the MELD score as an alternative to the DF score in predicting short-term mortality in persons with severe alcoholic hepatitis. Because many clinical laboratories now report the INR, and not the PT control values, the DF score cannot always be determined. Although the MELD score was not derived from patients with acute alcoholic hepatitis, it may be useful in selecting those individuals with alcoholic hepatitis who warrant specific interventions such as the use of pentoxyfilline or corticosteroids. 
Table I: Association of Clinical and Laboratory variables with 30-day mortality in patients with alcoholic hepatitis.

\begin{tabular}{|c|c|c|c|c|}
\hline Variable & & Number of patients & 30-day mortality, \% & p Value \\
\hline \multirow[t]{2}{*}{ Age } & $<46.5$ & 17 & 6 & 0.09 \\
\hline & $>46.5$ & 17 & 35 & \\
\hline \multirow[t]{2}{*}{ Gender } & Female & II & 27 & 0.66 \\
\hline & Male & 23 & 17 & \\
\hline \multirow[t]{2}{*}{ Temperature } & $<99.4$ & 27 & 19 & 0.61 \\
\hline & $>99.4$ & 7 & 29 & \\
\hline \multirow[t]{2}{*}{ Abdominal Pain } & Yes & 13 & 23 & 1.00 \\
\hline & No & 21 & 19 & \\
\hline \multirow[t]{2}{*}{ Cirrhosis } & Yes & 19 & 32 & 0.1 \\
\hline & No & 15 & 7 & \\
\hline \multirow[t]{2}{*}{ Encephalopathy } & Yes & II & 45 & 0.02 \\
\hline & No & 23 & 9 & \\
\hline \multirow[t]{2}{*}{ Varices } & Yes & 11 & 27 & 0.66 \\
\hline & No & 23 & 17 & \\
\hline \multirow[t]{2}{*}{ Splenomegaly } & Yes & 9 & 33 & 0.35 \\
\hline & No & 25 & 16 & \\
\hline \multirow[t]{2}{*}{ Ascites } & Yes & 17 & 41 & $<0.01$ \\
\hline & No & 17 & 0 & \\
\hline \multirow[t]{4}{*}{ Bilirubin (mg/dL) } & $<2$ & 15 & 7 & $<0.01$ \\
\hline & $2-5$ & 10 & 10 & \\
\hline & $5-8$ & 1 & 0 & \\
\hline & $>8$ & 8 & 63 & \\
\hline \multirow[t]{3}{*}{ Albumin $(g / d L)$} & $<3$ & 13 & 38 & 0.02 \\
\hline & $3-4$ & 13 & 0 & \\
\hline & $>4$ & 1 & 0 & \\
\hline \multirow[t]{2}{*}{ Creatinine (mg/dL) } & $<1.5$ & 27 & 15 & 0.13 \\
\hline & $>1.5$ & 7 & 43 & \\
\hline \multirow[t]{3}{*}{ AST/ALT } & $<1.2$ & 3 & 0 & 0.12 \\
\hline & $1.2-1.6$ & 5 & 0 & \\
\hline & $>1.6$ & 17 & 27 & \\
\hline \multirow[t]{3}{*}{ INR } & $<1.5$ & 20 & 5 & 0.01 \\
\hline & $1.5-2.5$ & 12 & 42 & \\
\hline & $>1.6$ & 2 & 50 & \\
\hline \multirow[t]{2}{*}{ AST (U/L) } & $<100$ & 9 & 0 & 0.15 \\
\hline & $>100$ & 23 & 26 & \\
\hline \multirow[t]{2}{*}{$\operatorname{ALT}(\mathrm{U} / \mathrm{L})$} & $<100$ & 18 & 22 & 1.00 \\
\hline & $>100$ & 7 & 14 & \\
\hline \multirow[t]{2}{*}{ WBC $\left(\times 10^{9} / \mathrm{L}\right)$} & $<10$ & 24 & 13 & 0.07 \\
\hline & $>10$ & 9 & 44 & \\
\hline \multirow[t]{2}{*}{ Hemoglobin $(g / d L)$} & $<10$ & 10 & 20 & 1.00 \\
\hline & $>10$ & 23 & 22 & \\
\hline \multirow[t]{2}{*}{ Platelet count $(\times 109 / \mathrm{L})$} & $<50$ & 3 & 33 & 0.52 \\
\hline & $>50$ & 30 & 20 & \\
\hline
\end{tabular}

The serum creatinine level is a component of the MELD score, but not the DF score. Indeed, the serum creatinine is highly associated with increased mortality in persons with end-stage chronic liver disease or cirrhosis. However, univariate analysis did not identify an association between an elevated creatinine and short-term mortality in our patients. Since histopathological confirmation was not available for all our patients, it was not possible to ascertain whether the MELD score may be a more useful prognostic score in those persons with co-existing cirrhosis and alcoholic hepatitis.

A barrier to clinical use of the MELD score is the complexity of the formula used to derive the score. Although the formula can be calculated readily programmed into a handheld or other computer, many persons will need to use a calculator to derive the score. Thus, prognostic factors derived at the bedside and based on simple clinical or 
Table 2: Relationship between mortality and the presence of ascites and hyperbilirubinemia in persons with alcoholic hepatitis.

\begin{tabular}{cccc}
\hline Bilirubin $>8 \mathrm{mg} / \mathrm{dL}$ & Ascites & Number of patients & 30-day Mortality \\
\hline & & & \\
Yes & Yes & 6 & $83 \%$ \\
Yes & No & 2 & $0 \%$ \\
No & Yes & 11 & $18 \%$ \\
No & No & 15 & $0 \%$ \\
\hline
\end{tabular}

biochemical criteria are likely to be more widely adopted and used. The presence of ascites, encephalopathy, increased bilirubin, and increased INR were predictive of 30-day mortality in our patients. Indeed, the combination of a total bilirubin greater than $8 \mathrm{mg} / \mathrm{dL}$ and the presence of ascites was highly predictive of mortality and more specific than either the MELD or the DF scores.

\section{Conclusions}

Alcoholic hepatitis remains associated with a high mortality in hospitalized patients. The MELD score is an accurate predictor of short term mortality. A MELD score of greater than 11 or simple clinical criteria, such as the co-existence of ascites and a total bilirubin level greater than $8 \mathrm{gm} / \mathrm{dL}$ should prompt consideration of specific therapeutic interventions such as the use of corticosteroids or pentoxyfilline to reduce mortality.

\section{Competing interests}

None declared

\section{Acknowledgements}

Supported by the Scott and White Memorial Hospital, Scott, Sherwood and Brindley Foundation, and Grant DK02678 from the National Institutes of Health.

\section{References}

I. Menon KV, Gores G], Shah VH: Pathogenesis, diagnosis, and treatment of alcoholic liver disease. Mayo Clin Proc 200I, 76:1021-1029

2. Morgan MY: The treatment of alcoholic hepatitis. Alcohol Alcohol 1996, 31:117-134

3. Maddrey WC, Boitnott JK, Bedine MS, Weber FL Jr, Mezey E, White RI Jr: Corticosteroid therapy of alcoholic hepatitis. Gastroenterology 1978, 75:193-199

4. Akriviadis E, Botla R, Briggs W, Han S, Reynolds T, Shakil O: Pentoxifylline improves short-term survival in severe acute alcoholic hepatitis: a double-blind, placebo-controlled trial. Gastroenterology 2000, I 19:1637-1648

5. Kamath PS, Wiesner RH, Malinchoc M, Kremers W, Therneau TM, Kosberg CL, et al: A model to predict survival in patients with end-stage liver disease. Hepatology 200I, 33:464-470

6. Chedid A, Mendenhall CL, Gartside P, French SW, Chen T, Rabin L: Prognostic factors in alcoholic liver disease. VA Cooperative Study Group. Am J Gastroenterol I991, 86:210-216

7. Fujimoto $M$, Uemura M, Kojima H, Ishii $Y$, Ann T, Sakurai S, et al: Prognostic factors in severe alcoholic liver injury. Nara Liver Study Group. Alcohol Clin Exp Res 1999, 23:33S-38S
8. Orrego H, Israel Y, Blake JE, Medline A: Assessment of prognostic factors in alcoholic liver disease: toward a global quantitative expression of severity. Hepatology 1983, 3:896-905

9. McCullough AJ, O'Connor JF: Alcoholic liver disease: proposed recommendations for the American College of Gastroenterology. Gastroenterol 1998, 93:2022-2036
Publish with BioMed Central and every scientist can read your work free of charge

"BioMedcentral will be the most significant development for disseminating the results of biomedical research in our lifetime." Paul Nurse, Director-General, Imperial Cancer Research Fund

Publish with BMC and your research papers will be:

- available free of charge to the entire biomedical community

- peer reviewed and published immediately upon acceptance

- cited in PubMed and archived on PubMed Central

- yours - you keep the copyright

Submit your manuscript here:

http://www.biomedcentral.com/manuscript/
BioMedcentral.com editorial@biomedcentral.com 